\title{
Pacific
}

Journal of

Mathematics

\section{HEEGAARD GENERA IN CONGRUENCE TOWERS OF HYPERBOLIC 3-MANIFOLDS}

\author{
BOGWANG JEON
}




\title{
HEEGAARD GENERA IN CONGRUENCE TOWERS OF HYPERBOLIC 3-MANIFOLDS
}

\author{
BOGWANG JEON
}

\begin{abstract}
Given a closed hyperbolic 3-manifold $M$, we construct a tower of covers with increasing Heegaard genus and give an explicit lower bound on the Heegaard genus of such covers as a function of their degree. Using similar methods, we prove that for any $\epsilon>0$ there exist infinitely many congruence covers $\left\{M_{i}\right\}$ such that, for any $x \in M, M_{i}$ contains an embedded ball $B_{x}$ $($ with center $x)$ satisfying $\operatorname{vol} B_{x}>\left(\operatorname{vol} M_{i}\right)^{1 / 4-\epsilon}$. We get similar results for an arithmetic noncompact case.
\end{abstract}

\section{Introduction}

Let $M$ be a hyperbolic 3-manifold and $\left\{M_{i}\right\}$ a collection of finite covers of $M$. The infimal Heegaard gradient of $M$ with respect to $\left\{M_{i}\right\}$ is defined as

$$
\inf _{i} \frac{\chi_{-}^{h}\left(M_{i}\right)}{\left[\pi_{1}(M): \pi_{1}\left(M_{i}\right)\right]},
$$

where $\chi_{-}^{h}\left(M_{i}\right)$ denotes the minimal value for the negative of the Euler characteristic of a Heegaard surface in $M_{i}$.

A fundamental question is whether the infimal Heegaard gradient is zero or not. This question is closely related to the potential solutions of several important conjectures in 3-manifold theory, such as the virtual Haken conjecture and the virtual fibering conjecture [Lackenby 2006; 2011]. Assuming the Lubotzky-Sarnak conjecture, a closed hyperbolic 3-manifold $M$ has a tower $\left\{M_{i}\right\}$ of finite covers without Property $\tau$. By a theorem of Lackenby [2006], if the infimal Heegaard gradient of this tower is positive, $M_{i}$ is Haken for sufficiently large $i$. According to a conjecture of Lackenby [2006], if the infimal Heegaard gradient of this tower is zero, $M_{i}$ is fibered for some $i$. Thus the Heegaard gradient plays an important role in these approaches to the virtual Haken conjecture and the virtual fibering conjecture.

When the manifold is arithmetic, Lackenby proved the following.

The author was partially supported by US NSF grant DMS-0707136.

MSC2010: primary 57M10, 57M27; secondary 11R04.

Keywords: Heegaard genus, hyperbolic 3-manifold, congruence cover. 
Theorem 1.1 [Lackenby 2006]. Let $M$ be an arithmetic hyperbolic 3-manifold. Then there are positive constants $c$ and $C$ which depend only on $M$, such that for any congruence cover $M_{i} \rightarrow M$,

$$
c\left[\pi_{1}(M): \pi_{1}\left(M_{i}\right)\right] \leq \chi_{-}^{h}\left(M_{i}\right) \leq C\left[\pi_{1}(M): \pi_{1}\left(M_{i}\right)\right] .
$$

He established this theorem by proving that Property $\tau$ with respect to a set of finite covers $\left\{M_{i}\right\}$ implies that $\left\{M_{i}\right\}$ has positive infimal Heegaard gradient. Since Lubotzky [1994] showed that an arithmetic hyperbolic 3-manifold has Property $\tau$ with respect to its congruence covers, Theorem 1.1 follows.

As we've seen, Heegaard genera and degrees of towers of covers provide important information and have strong connections with various things like Property $\tau$, but, unfortunately, little has been known about these in general [Lackenby 2006; Long et al. 2008]. Here, we construct towers of finite covers of hyperbolic 3manifolds with increasing Heegaard genera. While we do not show that the infimal Heegaard gradient is positive, we do give quantitative lower bounds for the Heegaard genus in terms of the degree of the cover. More precisely, we prove the following statement.

Theorem 1.2. Let $M$ be a closed hyperbolic 3-manifold and $\epsilon>0$ any (small) number. Then there exists a tower of finite congruence covers

$$
\cdots \rightarrow M_{i} \rightarrow \cdots \rightarrow M_{2} \rightarrow M
$$

such that the Heegaard genus of each $M_{i}$ is at least $\left[\pi_{1}(M): \pi_{1}\left(M_{i}\right)\right]^{\frac{1}{8}-\epsilon}$. If $M$ is arithmetic, we can improve the exponent $\frac{1}{8}-\epsilon$ to $\frac{1}{4}-\epsilon$.

For the arithmetic noncompact case, we get a similar result to the above arithmetic closed case.

Theorem 1.3. For a given arithmetic noncompact hyperbolic 3-manifold $M$ and any $\epsilon>0$, there exists a tower of finite congruence covers

$$
\cdots \rightarrow M_{i} \rightarrow \cdots \rightarrow M_{2} \rightarrow M
$$

such that the Heegaard genus of $M_{i}$ is at least $\geq\left[\pi_{1}(M): \pi_{1}\left(M_{i}\right)\right]^{\frac{1}{4}-\epsilon}$.

Although these results are weaker than Theorem 1.1 in the arithmetic case, our proofs involve different methods. In particular they use the result of Bachman, Cooper, and White about the relation between the injectivity radius and the Heegaard genus of a hyperbolic 3-manifold (see Theorem 2.1 and Corollary 2.2). Later, in Section 8, we analyze the limitations of these methods. It turns out that methods qualitatively similar to our own cannot prove analogues of Theorem 1.2 and Theorem 1.3 with $\frac{1}{8}-\epsilon$ and $\frac{1}{4}-\epsilon$ replaced by $x$ for any $x>\frac{1}{2}$. The proofs of Theorem 1.2 and Theorem 1.3 are similar in spirit but different in the details, so we give them separately. In addition, we prove the following theorems using 
the tools in the proofs of Theorem 1.2 and Theorem 1.3; see Section 2 for the definition of the lower injectivity radius, the principal congruence subgroup, and the Hecke-type congruences subgroups.

Theorem 1.4. For a given closed hyperbolic 3-manifold $M$ and for any $\epsilon>0$, there exist infinitely many congruence covers $\left\{M_{i}\right\}$ such that

$$
e^{r_{i}}>\left[\pi(M): \pi\left(M_{i}\right)\right]^{\frac{1}{8}-\epsilon}
$$

holds for all $i$, where $r_{i}$ is the lower injectivity radius of $M_{i}$. In addition, for any $x \in M_{i}, M_{i}$ contains an embedded ball $B_{x}$ with center $x$ and such that

$$
\operatorname{vol} B_{x}>\left(\operatorname{vol} M_{i}\right)^{\frac{1}{4}-\epsilon} \text {. }
$$

If $M$ is arithmetic, then we can improve the exponents $\frac{1}{8}-\epsilon$ and $\frac{1}{4}-\epsilon$ to $\frac{1}{4}-\epsilon$ and $\frac{1}{2}-\epsilon$, respectively.

Theorem 1.5. For a given arithmetic noncompact hyperbolic 3-manifold $M$, let $M^{\prime}$ be a finite cover of $M$ such that its fundamental group $\Gamma^{\prime}$ is a subgroup of a Bianchi group $\mathrm{PSL}_{2}\left(\mathrm{O}_{n}\right)$, and let I be a square-free ideal of $\mathrm{O}_{n}$ with no prime factors from a fixed finite set of prime ideals which depends only on $\Gamma^{\prime}$. Then the following statements hold.

(i) For any $\epsilon>0$, there exists $d>0$ depending on $\epsilon$ and $\Gamma^{\prime}$ such that if $M_{0}^{\prime}(I)$ is a cover induced by a Hecke-type congruence subgroup $\Gamma_{0}^{\prime}(I)$ with

$$
\left[\Gamma^{\prime}: \Gamma_{0}^{\prime}(I)\right]>d,
$$

$M_{0}^{\prime}(I)$ contains an embedded ball $B$ which satisfies

$$
\operatorname{vol} B>\left(\operatorname{vol} M_{0}^{\prime}(I)\right)^{\frac{1}{2}-\epsilon} .
$$

(ii) There exists $d>0$ depending only on $\Gamma^{\prime}$ such that if $M_{1}^{\prime}(I)$ is a cover induced by a Hecke-type congruence subgroup $\Gamma_{1}^{\prime}(I)$ with $\left[\Gamma^{\prime}: \Gamma_{1}^{\prime}(I)\right]>d, M_{1}^{\prime}(I)$ contains an embedded ball $B$ which satisfies

$$
\operatorname{vol} B>c\left(\operatorname{vol} M_{1}^{\prime}(I)\right)^{\frac{1}{4}} .
$$

(iii) There exists $d>0$ depending only on $\Gamma^{\prime}$ such that if $M^{\prime}(I)$ is a cover induced by a principal congruence subgroup $\Gamma^{\prime}(I)$ with $\left[\Gamma^{\prime}: \Gamma^{\prime}(I)\right]>d, M^{\prime}(I)$ contains an embedded ball $B$ which satisfies

$$
\operatorname{vol} B>c\left(\operatorname{vol} M^{\prime}(I)\right)^{\frac{1}{3}} .
$$

In fact, Theorem 1.5(iii) is shown in [Yeung 1994] with the better exponent 2/3 in a different way. 
Outline of the paper. In Section 2, we review some basic facts which we use in the proofs. We prove Theorems 1.2 and 1.4 in Sections 3 and 4, and Theorem 1.3 in Sections 5 and 6. In Section 7, we show Theorem 1.5. Finally, in Section 8 we briefly analyze why our method falls short of proving Theorem 1.1.

\section{Some background}

2A. Congruence subgroups. Let $\Gamma$ be the fundamental group of a finite-volume hyperbolic 3-manifold $M$ as a subgroup of $\mathrm{PSL}_{2}(\mathbb{C})\left(\cong \mathrm{SL}_{2}(\mathbb{C}) /\{ \pm I\}\right)$. Then, after conjugating, we can assume that there exists an embedding

$$
\rho: \Gamma \hookrightarrow \mathrm{PSL}_{2}\left(\mathrm{O}_{S}\right),
$$

where $O_{S}$ is the $S$-integers of an algebraic number field $K$ [Maclachlan and Reid 2003, Theorem 3.2.8] (taking $S$ to be the multiplicative set of the denominators of the generators of $\Gamma$ ). Given an ideal $J_{S}$ in $O_{S}$, the principal congruence subgroup of level $J_{S}$ of the group $\Gamma$ is the kernel of the natural reduction

$$
\rho_{J_{S}}: \Gamma \rightarrow \mathrm{PSL}_{2}\left(\mathrm{O}_{S} / J_{S}\right)
$$

and is denoted by $\Gamma\left(J_{S}\right)$. If $J_{S}=P_{1} \ldots P_{r}$ is a square free ideal of $O_{S}$ (so the $P_{i}$ are distinct prime ideals of $\mathrm{O}_{S}$ ), then, by the Chinese remainder theorem, we have

$$
\mathrm{SL}_{2}\left(O_{S} / J_{S}\right)=\mathrm{SL}_{2}\left(O_{S} / P_{1}\right) \times \cdots \times \mathrm{SL}_{2}\left(O_{S} / P_{r}\right) .
$$

Since

$$
\left|\mathrm{SL}_{2}\left(\mathrm{O}_{S} / P_{i}\right)\right|=\mathrm{N}\left(P_{i}\right)\left(\mathrm{N}^{2}\left(P_{i}\right)-1\right)
$$

for each prime ideal $P_{i}$, where $\mathrm{N}\left(P_{i}\right)$ is the norm of an ideal $P_{i}$ in $\mathrm{O}_{S}$, we get

$$
\left|\mathrm{PSL}_{2}\left(\mathrm{O}_{S} / J_{S}\right)\right|=\frac{1}{2} \prod_{i=1}^{r} \mathrm{~N}\left(P_{i}\right)\left(\mathrm{N}^{2}\left(P_{i}\right)-1\right) .
$$

Clearly the degree $\left[\Gamma: \Gamma\left(J_{S}\right)\right]$ is also bounded by the above number.

More generally, a congruence subgroup of $\Gamma$ is a subgroup of $\Gamma$ which contains a principal congruence subgroup. Typical examples are the Hecke-type congruence subgroups $\Gamma_{0}\left(J_{S}\right)$ and $\Gamma_{1}\left(J_{S}\right)$, which are defined to be

$$
\begin{aligned}
& \Gamma_{0}\left(J_{S}\right)=\left\{\gamma \in \Gamma: \rho_{J_{S}}(\gamma)=\left(\begin{array}{ll}
* & * \\
0 & *
\end{array}\right)\right\}, \\
& \Gamma_{1}\left(J_{S}\right)=\left\{\gamma \in \Gamma: \rho_{J_{S}}(\gamma)=\left(\begin{array}{ll}
1 & * \\
0 & 1
\end{array}\right)\right\},
\end{aligned}
$$

where

$$
\left(\begin{array}{ll}
* & * \\
0 & *
\end{array}\right) \text { and }\left(\begin{array}{ll}
1 & * \\
0 & 1
\end{array}\right)
$$


are the matrix representations of the elements in $\operatorname{PSL}_{2}\left(\mathrm{O}_{S} / J_{S}\right)$. These groups can also be expressed more explicitly as

$$
\begin{aligned}
& \Gamma_{0}\left(J_{S}\right)=\left\{\left(\begin{array}{ll}
a & b \\
c & d
\end{array}\right) \in \widehat{\Gamma} \mid\left(\begin{array}{ll}
a & b \\
c & d
\end{array}\right) \equiv\left(\begin{array}{ll}
* & * \\
0 & *
\end{array}\right) \bmod J_{S}\right\} /\{ \pm I\}, \\
& \Gamma_{1}\left(J_{S}\right)=\left\{\left(\begin{array}{ll}
a & b \\
c & d
\end{array}\right) \in \widehat{\Gamma} \mid\left(\begin{array}{ll}
a & b \\
c & d
\end{array}\right) \equiv \pm\left(\begin{array}{ll}
1 & * \\
0 & 1
\end{array}\right) \bmod I_{S}\right\} /\{ \pm I\},
\end{aligned}
$$

where $\widehat{\Gamma}$ is the inverse image of $\Gamma$ in $\operatorname{SL}_{2}\left(O_{S}\right)$.

Now we look at two simple cases where the map in (2-2) is surjective. First, for a prime ideal $P$ of $O_{S}$, extend the map in (2-1) to

$$
\Gamma \hookrightarrow \operatorname{PSL}_{2}\left(K_{P}\right)
$$

where $K_{P}$ is the $P$-adic local field. Then this restricts to a map

$$
\Gamma \hookrightarrow \mathrm{PSL}_{2}\left(\mathrm{O}_{P}\right)
$$

where $O_{P}$ is the unique $p$-adic integers of $K_{P}$. If we consider the reduction map

$$
\Gamma \rightarrow \operatorname{PSL}_{2}\left(\mathscr{O}_{P} / \pi \mathscr{O}_{P}\right)
$$

of (2-4) where $\pi O_{P}$ is the unique maximal ideal of $O_{P}$, it is clear that the map in (2-5) is actually the same as the one in (2-2) when $J_{S}=P$. According to [Long and Reid 1998], this map in (2-5) is surjective for almost all prime ideals $P$ such that $P$ is a prime ideal factor of a rational prime that splits completely in $\mathscr{O}_{K}$. A second case where (2-2) is surjective comes when $\Gamma$ is a subgroup of a Bianchi group, that is, $\Gamma \subset \mathrm{PSL}_{2}\left(\mathbb{O}_{K}\right)$ where $\mathbb{O}_{K}$ is the ring of integers of an imaginary quadratic number field $K$. Under this assumption, by the strong approximation theorem [Weisfeiler 1984], $\Gamma$ is dense in $\mathrm{PSL}_{2}\left(O_{P}\right)$ for almost all prime ideals $P$. If we define a natural map

$$
\phi: \mathrm{PSL}_{2}\left(\mathrm{O}_{P}\right) \rightarrow \mathrm{PSL}_{2}\left(\mathbb{O}_{P} / \pi \mathscr{O}_{P}\right),
$$

then, using the fact that $\Gamma$ is dense in $\operatorname{PSL}_{2}\left(O_{P}\right)$, we can get the surjection

$$
\Gamma \rightarrow \operatorname{PSL}_{2}\left(\mathrm{O}_{P}\right) / \operatorname{ker} \phi
$$

As $O_{P} / \pi O_{P}$ is isomorphic to $O_{K} / P O_{K},(2-6)$ and (2-7) give the surjective map

$$
\Gamma \rightarrow \mathrm{PSL}_{2}\left(\mathrm{O}_{K} / \mathrm{PO}_{K}\right) \text {. }
$$

These examples are particularly important because in these cases it is possible to calculate the indices of the various congruence subgroups explicitly. For example, if $I_{S}=P_{1} \ldots P_{r}$ is a square free ideal of $\mathrm{O}_{S}$ such that the maps $\Gamma \rightarrow \mathrm{PSL}_{2}\left(\mathrm{O}_{S} / P_{i}\right)$ 
are surjective for all prime ideals $P_{i}$, the index of $\Gamma / \Gamma\left(I_{S}\right)$ is given by (2-3). Furthermore, under the same assumption, it can be shown that

$$
\left[\Gamma: \Gamma_{0}(I)\right]=\prod_{1}^{r}\left(\mathrm{~N}\left(P_{i}\right)+1\right)
$$

and

$$
\left[\Gamma: \Gamma_{1}(I)\right]=\frac{1}{2} \prod_{i=1}^{r}\left(\mathrm{~N}^{2}\left(P_{i}\right)-1\right)
$$

(see [Miyake 1989, Chapter 4] for details).

2B. Injectivity radius. The injectivity radius of a Riemannian manifold $M$ at a point $x \in M, \operatorname{inj}_{x}(M)$, is the largest radius for which the exponential map at $x$ is a diffeomorphism. The upper injectivity radius, $\overline{\operatorname{inj}}(M)$, is the supremum of $\operatorname{inj}_{x}(M)$ as $x$ varies over $M$, and the lower injectivity radius, $\operatorname{inj}(M)$, is the infimum of $\operatorname{inj}_{x}(M)$ as $x$ varies over $M$. In particular, when $M$ is hyperbolic, the upper injectivity radius of $M$ is equal to

$$
\frac{1}{2} \sup \left\{\min _{g \neq I, g \in \Gamma}\left(d_{\mathbb{\boxplus}^{3}}(x, g(x))\right): x \in \mathbb{M}^{3}\right\}
$$

where $\Gamma$ is the fundamental group of $M$. Moreover if $M$ is closed, the lower injectivity radius of $M$ has the same value as half of the shortest length of a closed geodesic of $M$.

Bachman, Cooper, and White proved the following theorem, which provides an important method for bounding the Heegaard genus in terms of the injectivity radius.

Theorem 2.1 [Bachman et al. 2004]. If $M$ is a closed hyperbolic 3-manifold and $r=\overline{\operatorname{inj}}(M)$,

$$
\text { Heegaard genus of } M \geq \frac{1}{2} \cosh r \text {. }
$$

Although the above theorem was proved for closed manifolds, using Dehn filling we can extend the theorem as follows (see [Rubinstein 2005] for a similar result).

Corollary 2.2. The above inequality holds for finite-volume noncompact hyperbolic 3-manifolds.

Proof. A finite-volume noncompact hyperbolic 3-manifold $M$ can be approximated as a geometric limit of closed manifolds, that is, $M=\lim M_{n}$ where $\left\{M_{n}\right\}$ are closed manifolds obtained by Dehn filling. If we define $g$ (respectively $g_{n}$ ) to be the Heegaard genus of $M$ (respectively $M_{n}$ ), the inequality $g \geq g_{n}$ is true for all $n$ because Dehn filling never increases the Heegaard genus. Let $r$ (respectively $r_{n}$ ) be the upper injectivity radius of $M$ (respectively $M_{n}$ ). Then there exists an $\epsilon>0$ such that the $\epsilon$-thick part $M_{[\epsilon, \infty)}$ of $M$ has the same upper injectivity radius as $M$. 
Moreover, we can choose a uniform $\epsilon>0$ so that this is true for all $M_{n}$. Because $M_{n[\epsilon, \infty)}$ is approximately isometric to $M_{[\epsilon, \infty)}$ as $n \rightarrow \infty$, we get $r=\lim _{n \rightarrow \infty} r_{n}$. Now Corollary 2.2 follows from this, $g \geq g_{n}$, and Theorem 2.1.

The above theorem and corollary will be applied to calculate lower bounds for Heegaard genera as we mentioned in Section 1. Specifically, we use $\operatorname{inj}(M)$ in the proof of Theorem 1.2 and $\overline{\operatorname{inj}}(M)$ in the proof of Theorem 1.3.

2C. Closed geodesics. Next we quickly review closed geodesics. A closed geodesic of a hyperbolic 3-manifold is always induced by a hyperbolic element of its fundamental group as an invariant axis. We can detect its length by the trace value of the corresponding hyperbolic element [Maclachlan and Reid 2003, Chapter 11]. Concerning the asymptotic number of closed geodesics of a given closed hyperbolic 3-manifold $M$ as a function of length, we have the following nice formula:

Prime geodesic theorem [Margulis 1969]. For a closed hyperbolic 3-manifold $M$, the number of primitive elements of length less than or equal to $l$ is asymptotic to $e^{2 l} / 2 l$ as $l$ goes to infinity.

Here a primitive element of $\Gamma$ is one that is not a nontrivial power of any element in $\Gamma$. If we denote by \# $(l)$ the number of closed geodesics of length less than or equal to $l$ in $M$, we can get the upper bound of \#(l) using the prime geodesic theorem.

Corollary 2.3. Suppose that $M, \Gamma$, and \#(l) are the same as above. Then there exists a constant $c^{\prime}$ depending only on $\Gamma$ such that

$$
\#(l)<c^{\prime} e^{2 l} .
$$

Proof. Put $\#_{\text {prm }}(l)$ the number of primitive elements of length less than or equal to $l$. Then, by the prime geodesic theorem, there exists $d>0$ such that

$$
\#_{\mathrm{prm}}(l)<\frac{e^{2 l}}{l}
$$

for all $l>d$. Since every hyperbolic element $h \in \Gamma$ is of the form $g^{m}$ where $g$ is a primitive element in $\Gamma$ and $m \in \mathbb{N}$, we have

$$
\#(l)=\sum_{i=1}^{\infty} \#_{\mathrm{prm}}(l / i) .
$$

Let $s$ be the length of a shortest geodesic of $M$. Because $\#_{\text {prm }}(l / i)=0$ for $i>\lfloor l / s\rfloor$, we can rewrite (2-12) as

$$
\#(l)=\sum_{i=1}^{\infty} \#_{\mathrm{prm}}(l / i)=\sum_{i=1}^{\lfloor l / s\rfloor} \#_{\mathrm{prm}}(l / i) .
$$


Clearly $\#_{\mathrm{prm}}(l / i) \leq \#_{\mathrm{prm}}(l)$. Thus, if $l>d$, $\#_{\mathrm{prm}}(l / i)<e^{2 l} / l$ for $1 \leq i \leq\lfloor l / s\rfloor$. Combining this with (2-13), we get

$$
\#(l)<\lfloor l / s\rfloor \frac{e^{2 l}}{l} \leq \frac{e^{2 l}}{s}
$$

for $l>d$. If we take $c^{\prime}>0$ bigger than \#(d) and $1 / s, \#(l)<c^{\prime} e^{2 l}$ for all $l>0$.

2D. Number theory. Lastly we quote two important theorems from number theory and deduce two corollaries of them.

Prime number theorem [Bateman and Diamond 2004]. Let $\pi(x)$ be the number of rational primes which are less than or equal to $x$. Then $\pi(x)$ is asymptotic to $x / \log x$ as $x$ goes to infinity and we denote this by

$$
\pi(x) \sim \frac{x}{\log x} .
$$

In addition, this is equivalent to

$$
\theta(x) \sim x,
$$

where $\theta(x)=\sum_{p<x} \log p$.

Chebotarev's density theorem [Narkiewicz 2004]. Let $K / \mathbb{Q}$ be a number field and $L$ be the Galois closure of $K$. If $S_{1}$ denotes the set of all primes of $\mathbb{Z}$ which split completely over $K$, the following inequality holds.

$$
\liminf _{x \rightarrow \infty} \frac{\#\left\{p \in S_{1}: p \leq x\right\}}{\#\{p: p \leq x\}} \geq \frac{1}{n}
$$

where $n=[L: \mathbb{Q}]$.

Corollary 2.4. With the same notations as in the above theorem, we can find a subset $S_{2}$ of $S_{1}$ such that

$$
\lim _{x \rightarrow \infty} \frac{\#\left\{p \in S_{2}: p \leq x\right\}}{\#\{p: p \leq x\}}=\frac{1}{n},
$$

that is,

$$
\lim _{x \rightarrow \infty} \frac{\pi_{S_{2}}(x)}{x / \log x}=\frac{1}{n},
$$

where $\pi_{S_{2}}(x)$ is the number of primes of $S_{2}$ which are less than or equal to $x$. Furthermore, the above formulas are equivalent to

$$
\lim _{x \rightarrow \infty} \frac{\theta_{S_{2}}(x)}{x}=\frac{1}{n},
$$

where $\theta_{S_{2}}(x)=\sum_{p<x, p \in S_{2}} \log p$. 
Proof. From

$$
\liminf _{x \rightarrow \infty} \frac{\#\left\{p \in S_{1}: p \leq x\right\}}{\#\{p: p \leq x\}} \geq \frac{1}{n},
$$

the first result follows. The second argument can be deduced by copying the analogous steps of the proof of the equivalence of $\pi(x) \sim x / \log x$ and $\theta(x) \sim x$; for example, see [Bateman and Diamond 2004, Chapter 4].

Corollary 2.5. With the same notations as in Corollary 2.4, let $p_{k}$ be the $k$-th prime number in $S_{2}$ and $d_{k}=p_{1} \ldots p_{k}$. Then, for sufficiently large $k, p_{k+1}$ is less than $2 n \log d_{k}$, so, for any sufficiently large natural number $x$, there exists a prime number $p \in S_{2}$ such that $p \nmid x$ and $p<2 n \log x$.

Proof. This immediately follows from the definition of $\theta_{S_{2}}(x)$ and the formula

$$
\lim _{x \rightarrow \infty} \frac{\theta_{S_{2}}(x)}{x}=\frac{1}{n}
$$

\section{Proof of Theorems 1.2 and 1.4}

Throughout Sections 3 and $4, \Gamma$ is the fundamental group of the closed hyperbolic 3-manifold $M$ and $\widehat{\Gamma}$ is the inverse image of $\Gamma$ in $\operatorname{SL}_{2}\left(O_{S}\right)$. We also denote the two inverse images of $\gamma \in \Gamma$ in $\widehat{\Gamma}$ by $\pm \hat{\gamma}$.

Proof of Theorem 1.2. We start by sketching the key idea of the proof. For a given closed geodesic of length less than or equal to $l$, using the facts that a closed geodesic is always induced by a hyperbolic element and that \# $(l)$ is finite, we find a prime ideal $P\left(\right.$ of $\left.O_{S}\right)$ such that its principal congruence group $\Gamma(P)$ doesn't contain any hyperbolic elements of length less than or equal to $l$. Then, applying Theorem 2.1 and formula (2-10), we calculate bounds for the Heegaard genus and the index of $\Gamma(P)$. The next lemma, which we'll prove in the next section, is important for calculating these bounds.

Lemma 3.1. For $\omega \in \Gamma$ of hyperbolic length less than or equal to $l$, there exists $\alpha, \beta \in \mathfrak{O}_{K}$ such that $\pm \operatorname{tr} \hat{\omega}= \pm \alpha / \beta$ and

$$
|\mathrm{N}(\alpha \pm 2 \beta)|<\left(C_{3}\right)^{l},
$$

where $C_{3} \geq 1$ is a constant which depends only on $\Gamma$.

The proof of the lemma is not difficult but it involves some preliminaries, so we'll prove it independently in Section 4. Here, using the lemma, we prove the following claim.

Lemma 3.2. For any $d>0$, there exists a Hecke-type congruence subgroup $\Gamma_{1}\left(P_{S}\right)$ such that $\left[\Gamma: \Gamma_{1}\left(P_{S}\right)\right]>d$ and

$$
\text { Heegaard genus of } M_{1}\left(P_{S}\right) \geq\left[\Gamma: \Gamma_{1}\left(P_{S}\right)\right]^{\frac{1}{8}-\epsilon / 2},
$$


where $M_{1}\left(P_{S}\right)$ is the cover induced by $\Gamma_{1}\left(P_{S}\right)$ and $\epsilon>0$ is any small number. Proof. First, let $l>0$ be an arbitrary number and

$$
\left\{ \pm \operatorname{tr} \hat{\omega}_{1}, \pm \operatorname{tr} \hat{\omega}_{2}, \ldots, \pm \operatorname{tr} \hat{\omega}_{r(l)}\right\}
$$

the set of traces of images in $\widehat{\Gamma}$ of all hyperbolic elements of length less than or equal to $l$. Then, by Corollary $2.3, r(l) \leq \#(l)<c^{\prime} e^{2 l}$ for some constant $c^{\prime}$ which depends only on $\Gamma$. Now Lemma 3.1 implies that, for each $i$, we can find $\alpha_{i}, \beta_{i} \in \mathscr{O}_{K}$ with $\pm \operatorname{tr} \hat{\omega}_{i}= \pm \alpha_{i} / \beta_{i}$ such that

$$
\prod_{i=1}^{r(l)}\left|\mathrm{N}\left(\alpha_{i}-2 \beta_{i}\right)\right|\left|\mathrm{N}\left(\alpha_{i}+2 \beta_{i}\right)\right| \leq\left(C_{3}^{2 l}\right)^{r(l)} \leq\left(C_{3}^{2 l}\right)^{c^{\prime} e^{2 l}} .
$$

Claim 3.3. If $l \rightarrow \infty, r(l) \rightarrow \infty$ and

$$
\prod_{i=1}^{r(l)}\left|\mathrm{N}\left(\alpha_{i}-2 \beta_{i}\right)\right|\left|\mathrm{N}\left(\alpha_{i}+2 \beta_{i}\right)\right| \rightarrow \infty .
$$

Proof. Suppose

$$
\prod_{i=1}^{r(l)}\left|\mathrm{N}\left(\alpha_{i}-2 \beta_{i}\right)\right|\left|\mathrm{N}\left(\alpha_{i}+2 \beta_{i}\right)\right|
$$

is bounded as $l \rightarrow \infty$. Pick a rational prime $p$ such that $p$ doesn't divide the norm of any generators of $S$ and

$$
p>\prod_{i=1}^{r(l)}\left|\mathrm{N}\left(\alpha_{i}-2 \beta_{i}\right)\right|\left|\mathrm{N}\left(\alpha_{i}+2 \beta_{i}\right)\right|
$$

for all $l$. If $P$ is a prime factor of $p 0_{K}$,

$$
\mathrm{N}(P) \nmid \prod_{i=1}^{r(l)}\left|\mathrm{N}\left(\alpha_{i}-2 \beta_{i}\right)\right|\left|\mathrm{N}\left(\alpha_{i}+2 \beta_{i}\right)\right|,
$$

so, for all $i$,

$$
\alpha_{i} \pm 2 \beta_{i} \notin P \Rightarrow\left(\alpha_{i} \pm 2 \beta_{i}\right) / \beta_{i}=\operatorname{tr} \hat{\omega}_{i} \pm 2 \notin P_{S} \Rightarrow \omega_{i} \notin \Gamma_{0}\left(P_{S}\right),
$$

where $\Gamma_{0}\left(P_{S}\right)$ is a Hecke-type congruence subgroup of $\Gamma$. This implies $\Gamma_{0}\left(P_{S}\right)$ doesn't contain any elements of $\Gamma$, contradicting the fact that $\Gamma_{0}\left(P_{S}\right)$ is a finite index subgroup of $\Gamma$.

By the above claim and Corollary 2.5, if $l$ is sufficiently large, there exists a prime $p$ such that

$$
p \nmid \prod_{i=1}^{r(l)}\left|\mathrm{N}\left(\alpha_{i}-2 \beta_{i}\right)\right|\left|\mathrm{N}\left(\alpha_{i}+2 \beta_{i}\right)\right|,
$$


$p$ splits completely over $K$, and

$$
p<2 n \log \left(\prod_{i=1}^{r(l)}\left|\mathrm{N}\left(\alpha_{i}-2 \beta_{i}\right)\right|\left|\mathrm{N}\left(\alpha_{i}+2 \beta_{i}\right)\right|\right)<2 n \log \left(C_{3}^{2 c^{\prime} l e^{2 l}}\right),
$$

where $n$ is the degree of the Galois closure of $K$ as was previously defined in Section 2D. Define $p(l)$ to be the smallest prime which satisfies the above conditions for the given $l$. Then, by the same reasoning as in the proof of Claim 3.3, we have $p(l) \rightarrow \infty$ as $l \rightarrow \infty$.

Now let's assume $l$ is sufficiently large so that $p(l)$ doesn't divide the norm of any generators of $S$ and any prime factor of $p(l) \mathcal{O}_{S}$ gives a surjection in (2-2). Set $P_{S}$ to be one of the prime factors of $p(l) 0_{S}, \Gamma_{1}\left(P_{S}\right)=$ a Hecke-type congruence subgroup, and $M_{1}\left(P_{S}\right)=$ the corresponding cover of $\Gamma_{1}\left(P_{S}\right)$. Since $P_{S}$ satisfies (3-2) and (3-3), the length of a shortest closed geodesic of $\Gamma_{1}\left(P_{S}\right)$ is bigger than $l$, so

$$
\text { Heegaard genus of } M_{1}\left(P_{S}\right) \geq \frac{1}{4} e^{l / 2} \text {. }
$$

As $\mathrm{N}\left(P_{S}\right)$ is equal to $p(l)$, the degree $\left[\Gamma: \Gamma_{1}\left(P_{S}\right)\right]$ is less than

$$
\frac{1}{2}(p(l))^{2}
$$

by $(2-10)$, and thus,

$$
\frac{1}{2}(p(l))^{2}<\frac{1}{2}\left(2 n \log \left(C_{3}^{2 c^{\prime} l e^{2 l}}\right)\right)^{2}=2 n^{2} \log ^{2}\left(C_{3}^{2 c^{\prime} l e^{2 l}}\right)=8 n^{2}\left(c^{\prime} l e^{2 l}\right)^{2} \log ^{2} C_{3},
$$

because $p(l)<2 n \log \left(C_{3}^{2 c^{\prime} l e^{2 l}}\right)$. Now it is easy to check that, for any $\epsilon>0$,

$$
\frac{e^{l / 2}}{4} \geq\left(8 n^{2}\left(c^{\prime} l e^{2 l}\right)^{2} \log ^{2} C_{3}\right)^{\frac{1}{8}-\epsilon / 2}
$$

for sufficiently large $l$. This means

$$
\text { Heegaard genus of } M_{1}\left(P_{S}\right) \geq\left[\Gamma: \Gamma_{1}\left(P_{S}\right)\right]^{\frac{1}{8}-\epsilon / 2} .
$$

From the construction, it is clear that we can make $M_{1}\left(P_{S}\right)$ with arbitrary large degree and Heegaard genus.

Note that, in the proof of Lemma 3.2, we actually showed

$$
\text { Heegaard genus of } M_{1}\left(P_{S}\right) \geq \frac{e^{t / 2}}{4} \geq\left(\frac{1}{2}\left(\mathrm{~N}\left(P_{S}\right)\right)^{2}\right)^{\frac{1}{8}-\epsilon / 2},
$$

where $t$ is the length of a shortest closed geodesic in $M_{1}\left(P_{S}\right)$.

Now we go back to the proof of Theorem 1.2. Let's construct a tower of finite covers of $M$. First, consider a Hecke congruence subgroup $\Gamma_{1}\left(P_{1}\right)$ of a prime ideal 
$P_{1}$ of $O_{S}$ which satisfies the inequality (3-1) for a given $\epsilon>0$. Next pick a prime ideal $P_{2}$ (from Lemma 3.2) with $\mathrm{N}\left(P_{2}\right)$ sufficiently large such that it satisfies

$$
\left(\frac{1}{2}\left(\mathrm{~N}\left(P_{2}\right)\right)^{2}\right)^{\frac{1}{8}-\epsilon / 2}>\left(\frac{1}{2}\left(\mathrm{~N}\left(P_{1} P_{2}\right)\right)^{2}\right)^{\frac{1}{8}-\epsilon} .
$$

If $M\left(P_{1} P_{2}\right)$ (respectively $\left.M\left(P_{2}\right)\right)$ denotes a corresponding manifold of a Hecketype congruence subgroup $\Gamma_{1}\left(P_{1} P_{2}\right)$ (respectively $\Gamma_{1}\left(P_{2}\right)$ ), the length of a shortest closed geodesic in $M_{1}\left(P_{1} P_{2}\right)$ is bigger than the length of a shortest geodesic in $M\left(P_{2}\right)$, because $\Gamma_{1}\left(P_{1} P_{2}\right) \subset \Gamma_{1}\left(P_{2}\right)$. Thus, by (3-4), the Heegaard genus of $M_{1}\left(P_{1} P_{2}\right)$ is at least

$$
\left(\frac{1}{2}\left(\mathrm{~N}\left(P_{2}\right)\right)^{2}\right)^{\frac{1}{8}-\epsilon / 2} .
$$

Since the degree of $\Gamma_{1}\left(P_{1} P_{2}\right)$ is less than $\frac{1}{2}\left(\mathrm{~N}\left(P_{1} P_{2}\right)\right)^{2}$, from (3-5), we get

$$
\text { Heegaard genus of } M_{1}\left(P_{1} P_{2}\right) \geq\left[\Gamma: \Gamma_{1}\left(P_{1} P_{2}\right)\right]^{\frac{1}{8}-\epsilon} \text {. }
$$

By induction, for $n \geq 2$, let's pick a prime ideal $P_{n+1}$ having sufficiently large $\mathrm{N}\left(P_{n+1}\right)$ so that it satisfies

$$
\left(\frac{1}{2}\left(\mathrm{~N}\left(P_{n+1}\right)\right)^{2}\right)^{\frac{1}{8}-\epsilon / 2}>\left(\frac{1}{2}\left(\mathrm{~N}\left(P_{1} \ldots P_{n+1}\right)\right)^{2}\right)^{\frac{1}{8}-\epsilon} .
$$

Define $M_{1}\left(P_{1} \ldots P_{n+1}\right)$ to be the corresponding cover of the Hecke-type congruence subgroup $\Gamma_{1}\left(P_{1} \ldots P_{n+1}\right)$. Then, by (2-10), the degree of $\Gamma_{1}\left(P_{1} \ldots P_{n+1}\right)$ is less than $\frac{1}{2}\left(\mathrm{~N}\left(P_{1} \ldots P_{n}\right)\right)^{2}$, but the Heegaard genus of $M_{1}\left(P_{1} \ldots P_{n+1}\right)$ is at least

$$
\left(\frac{1}{2}\left(\mathrm{~N}\left(P_{n+1}\right)\right)^{2}\right)^{\frac{1}{8}-\epsilon / 2},
$$

by the same reasoning as above. Hence

Heegaard genus of $\left.M_{1}\left(P_{1} \ldots P_{n+1}\right) \geq\left[\Gamma: \Gamma_{1}\left(P_{1} \ldots P_{n+1}\right)\right)\right]^{\frac{1}{8}-\epsilon}$.

Now, $\Gamma_{1}\left(P_{1}\right), \Gamma_{1}\left(P_{1} P_{2}\right), \ldots$ gives a desired sequence of congruence covers for Theorem 1.2. This completes the proof of Theorem 1.2 for the general case.

We now prove the arithmetic case. If $M$ is arithmetic, then there exists a cover $M^{\prime}$ with fundamental group $\Gamma^{\prime}$ such that $\Gamma^{\prime}$ is a subgroup of a maximal order of a quaternion algebra; see [Maclachlan and Reid 2003, Chapter 8]. In this case, it is proved in [Marklof 1996] that the number of distinct complex lengths of real length less than or equal to $l$ in $M^{\prime}$ is bounded by $c^{\prime \prime} e^{l}$, where $c^{\prime \prime}$ is a constant depending only on $M^{\prime}$. Applying the bound $c^{\prime \prime} e^{l}$ instead of $c^{\prime} e^{2 l}$, we can check that Lemma 3.2 and the above construction of a sequence of covers still hold for $M^{\prime}$ with the reduced exponents $\frac{1}{4}-\epsilon / 2$ and $\frac{1}{4}-\epsilon$, instead of $\frac{1}{8}-\epsilon / 2, \frac{1}{8}-\epsilon$, respectively. In other words, for any $\epsilon>0, M^{\prime}$ has a sequence of congruence covers $\left\{\Gamma_{i}\right\}$ such that

Heegaard genus of the cover induced by $\Gamma_{i} \geq\left[\Gamma: \Gamma_{i}\right]^{\frac{1}{4}-\epsilon}$ 
with arbitrarily large $\left[\Gamma: \Gamma_{i}\right]$ for each $i$.

Using this, we show that $M$ has the same property. For any $\epsilon>0$, first pick $\epsilon^{\prime}>0$ that is smaller than $\epsilon$ and construct a tower of Hecke-type congruence subgroups $\left\{\Gamma_{i}^{\prime}\right\}$ of $\Gamma^{\prime}$ that satisfies

$$
\left[\Gamma^{\prime}: \Gamma_{i}^{\prime}\right]^{\frac{1}{4}-\epsilon^{\prime}} \geq\left[\Gamma: \Gamma_{i}^{\prime}\right]^{\frac{1}{4}-\epsilon}=\left(\left[\Gamma: \Gamma^{\prime}\right]\left[\Gamma^{\prime}: \Gamma_{i}^{\prime}\right]\right)^{\frac{1}{4}-\epsilon}
$$

and

Heegaard genus of the cover induced by $\Gamma_{i}^{\prime} \geq\left(\left[\Gamma^{\prime}: \Gamma_{i}^{\prime}\right]\right)^{\frac{1}{4}-\epsilon^{\prime}}$

for each $i$. The existence of $\left\{\Gamma_{i}^{\prime}\right\}$ is guaranteed by the earlier discussion. Clearly $\left\{\Gamma_{i}^{\prime}\right\}$ satisfies

Heegaard genus of the cover induced by $\Gamma_{i}^{\prime} \geq\left(\left[\Gamma: \Gamma_{i}^{\prime}\right]\right)^{\frac{1}{4}-\epsilon}$.

This gives a desired tower of finite covers of $M$ for the given $\epsilon$.

Proof of Theorem 1.4. Let $\epsilon>0$ be fixed. In the proof of Lemma 3.2, we used the fact that

$$
\frac{e^{l / 2}}{4} \geq\left(8 n^{2}\left(c^{\prime} l e^{2 l}\right)^{2} \log ^{2} C_{3}\right)^{\frac{1}{8}-\epsilon / 2}
$$

holds for sufficiently large $l$. Changing the inequality slightly so that, for any given $0<c_{1}<\pi / 2$, if we write

$$
e^{l / 2} \geq\left(8 n^{2}\left(c^{\prime} l e^{2 l}\right)^{2} \log ^{2} C_{3}\right)^{\frac{1}{8}-\epsilon / 2}\left(\geq\left[\Gamma: \Gamma_{1}\left(P_{S}\right)\right]^{\frac{1}{8}-\epsilon / 2}\right)
$$

and

$$
c_{1} e^{l} \geq\left(\operatorname{vol} M 8 n^{2}\left(c^{\prime} l e^{2 l}\right)^{2} \log ^{2} C_{3}\right)^{\frac{1}{4}-\epsilon}\left(\geq \operatorname{vol} M\left(P_{S}\right)^{\frac{1}{4}-\epsilon}\right),
$$

the above inequalities are also true for all sufficiently large $l$. The volume of a hyperbolic ball of radius $r$ is

$$
\pi(\sinh (2 r)-2 r)
$$

and it is bounded below by $c_{1} e^{2 r}$, when $r$ is sufficiently large. Now the result follows from this, (3-6), (3-7), and similar steps in the proof of Lemma 3.2. If $M$ is arithmetic, we can get the desired result by replacing the exponents $\frac{1}{8}-\epsilon / 2$ in (3-6) and $\frac{1}{4}-\epsilon$ in (3-7) with $\frac{1}{4}-\epsilon / 2$ and $\frac{1}{2}-\epsilon$, respectively.

\section{Proof of Lemma 3.1}

Let $R$ be a finite set of generators of $\Gamma$. The minimal word length of $\omega \in \Gamma$ is defined to be

$$
\min \left\{\left|t_{1}\right|+\cdots+\left|t_{k}\right|: \omega=\gamma_{1}^{t_{1}} \ldots \gamma_{k}^{t_{k}} \quad \text { and } \quad \gamma_{i} \in R \text { for } 1 \leq i \leq k\right\} .
$$


According to [Milnor 1968], since $M$ is compact, the Cayley graph of $\Gamma$ with respect to $R$ is quasi-isometric to its universal cover $\mathbb{M}^{3}$, so the minimal word length of $\omega$ is bounded by $c^{\prime} l$, where $l$ is the length of $\omega$ and $c^{\prime}$ is a positive constant which depends only on $\Gamma$.

Let $G$ be the finite set of Galois embeddings of the number field $K$ in $\mathbb{C}$, and

$$
C_{1}=\max \{1, \max \{|\sigma(a)|: a \text { is an entry of } \pm \hat{\gamma} \text { for any } \gamma \in R, \sigma \in G\}\} .
$$

Note that, for any $\gamma \in R$, the constant $C_{1}$ is also an upper bound for the absolute values of all Galois conjugates of all the entries of $\pm \hat{\gamma}^{-1}$, since the determinant of $\hat{\gamma}$ is equal to 1 . The following claim is important in the proof of Lemma 3.1.

Claim 4.1. If $k>0$ is the minimal word length of $\omega \in \Gamma$ with respect to the generating set $R$, and

$$
\pm \hat{\omega}= \pm\left(\begin{array}{ll}
\omega_{1} & \omega_{2} \\
\omega_{3} & \omega_{4}
\end{array}\right) \in \widehat{\Gamma}
$$

where $\omega_{j} \in \mathcal{O}_{S}$, then $\left|\sigma\left(\omega_{j}\right)\right|$ is bounded by $2^{k-1} C_{1}^{k}$ for all $j$ and $\sigma \in G$.

Proof. We induct on $k$. First, the case where $k=1$ is clear. Suppose $k \geq 2$ and the claim is true for all $i \leq k-1$. Since $k \geq 2, \omega$ has one of the forms $\gamma \omega^{\prime}$ or $\gamma^{-1} \omega^{\prime}$, where $\omega^{\prime}$ has word length $k-1$ and $\gamma \in R$. We will prove only the case $\omega=\gamma \omega^{\prime}$ because the other case is similar. If

$$
\pm \hat{\gamma}= \pm\left(\begin{array}{ll}
a & b \\
c & d
\end{array}\right) \quad \text { and } \quad \pm \hat{\omega}^{\prime}= \pm\left(\begin{array}{cc}
\omega_{1}^{\prime} & \omega_{2}^{\prime} \\
\omega_{3}^{\prime} & \omega_{4}^{\prime}
\end{array}\right)
$$

then

$$
\pm \hat{\omega}= \pm\left(\begin{array}{ll}
a & b \\
c & d
\end{array}\right)\left(\begin{array}{cc}
\omega_{1}^{\prime} & \omega_{2}^{\prime} \\
\omega_{3}^{\prime} & \omega_{4}^{\prime}
\end{array}\right)= \pm\left(\begin{array}{cc}
a \omega_{1}^{\prime}+b \omega_{3}^{\prime} & a \omega_{2}^{\prime}+b \omega_{4}^{\prime} \\
c \omega_{1}^{\prime}+d \omega_{3}^{\prime} & c \omega_{2}^{\prime}+d \omega_{4}^{\prime}
\end{array}\right)
$$

Focusing on the upper left entry of $\pm \hat{\omega}$, we have

$$
\left|\sigma\left(a \omega_{1}^{\prime}+b \omega_{3}^{\prime}\right)\right| \leq|\sigma(a)|\left|\sigma\left(\omega_{1}^{\prime}\right)\right|+|\sigma(c)|\left|\sigma\left(\omega_{3}^{\prime}\right)\right| .
$$

Since

$$
\left|\sigma\left(\omega_{1}^{\prime}\right)\right|,\left|\sigma\left(\omega_{3}^{\prime}\right)\right| \leq 2^{k-2} C_{1}^{k-1}
$$

for all $\sigma \in G$ by induction, we get

$$
\left|\sigma\left(\omega_{1}\right)\right| \leq\left(2^{k-2} C_{1}^{k-1}\right)\left(C_{1}\right)+\left(2^{k-2} C_{1}^{k-1}\right)\left(C_{1}\right)=2^{k-1} C_{1}^{k}
$$

for all $\sigma \in G$. The same estimate holds for the other entries of $\pm \hat{\omega}$.

Proof of Lemma 3.1. Let $\omega \in \Gamma$ have length at most $l$ as stated in the lemma. By Claim 4.1, $|\sigma( \pm \operatorname{tr} \hat{\omega})|$ is bounded by $2^{c^{\prime} l-1} C_{1}^{c^{\prime} l}+2^{c^{\prime} l-1} C_{1}^{c^{\prime} l}=2^{c^{\prime} l} C_{1}^{c^{\prime} l}$ for all $\sigma \in G$. Since $R$ is a finite set, we can find a common denominator $\beta^{\prime} \in \mathscr{O}_{K}$ of all the entries 
of images of elements of $R$ in $\operatorname{SL}_{2}\left(O_{S}\right)$. That is, there exists $\beta^{\prime} \in \mathrm{O}_{K}$ such that, for any $\gamma \in R, \pm \hat{\gamma}$ can be represented in the form

$$
\pm\left(\begin{array}{cc}
\alpha_{1} / \beta^{\prime} & \alpha_{2} / \beta^{\prime} \\
\alpha_{3} / \beta^{\prime} & \alpha_{4} / \beta^{\prime}
\end{array}\right)
$$

where the $\alpha_{i} \in \mathscr{O}_{K}$ depend on $\gamma$. If we put

$$
C_{2}=\max \left\{\left|\sigma\left(\beta^{\prime}\right)\right|: \sigma \in G\right\},
$$

then, as $\omega$ has word length at most $c^{\prime} l$, we get $\alpha, \beta \in \mathbb{O}_{K}$ such that $\pm \operatorname{tr} \hat{\omega}= \pm \alpha / \beta$ and $|\sigma(\beta)|$ is bounded by $C_{2}^{c^{\prime} l}$ for all $\sigma \in G$. Since

$$
|\sigma(\alpha \pm 2 \beta)|=|\sigma(\operatorname{tr} \hat{\omega}) \pm 2||\sigma(\beta)|,
$$

$|\sigma(\alpha \pm 2 \beta)|$ is bounded by

$$
\left(2^{c^{\prime} l} C_{1}^{c^{\prime} l}+2\right) C_{2}^{c^{\prime} l}=\left(2 C_{1} C_{2}\right)^{c^{\prime} l}+2 C_{2}^{c^{\prime} l} \leq 2\left(2 C_{1} C_{2}\right)^{c^{\prime} l}
$$

for all $\sigma \in G$. Because

$$
\mathrm{N}(\alpha \pm 2 \beta) \leq(\max \{|\sigma(\alpha \pm 2 \beta)|: \sigma \in G\})^{m},
$$

where $m=[K: \mathbb{Q}]$, we get

$$
\mathrm{N}(\alpha \pm 2 \beta) \leq 2\left(2 C_{1} C_{2}\right)^{c^{\prime} l m} .
$$

Since $l$ is the hyperbolic length of a closed manifold $M$, it is bounded below by the length $s$ of the shortest closed geodesic. Now it is straightforward to check that there exists $C_{3} \geq 1$ such that

$$
\left(C_{3}\right)^{l}>2\left(2 C_{1} C_{2}\right)^{c^{\prime} l m}
$$

for all $l \geq s$.

\section{A technical lemma for Theorem 1.3}

Now we embark on the proof of Theorem 1.3. The key idea of the proof is simpler than that of Theorem 1.2. We'll show that in the arithmetic noncompact case, the upper injectivity radius of a Hecke-type congruence subgroup is always bounded by a function of its degree. First, recall that if $M$ is an arithmetic noncompact manifold, it has a finite cover $M^{\prime}$ such that its fundamental group $\Gamma^{\prime}$ is a subgroup of a Bianchi group $\mathrm{PSL}_{2}\left(\mathrm{O}_{n}\right)$ where $\mathrm{O}_{n}$ is an imaginary quadratic number field [Maclachlan and Reid 2003, Chapter 8]. The following lemma will provide a way to get a lower bound of upper injectivity radii of the congruence subgroups of $\Gamma^{\prime}$. Although the hypotheses of the lemma may seem artificial, they are satisfied for the congruence subgroups (of $\Gamma^{\prime}$ ) under consideration, as we explain after the proof. 
Lemma 5.1. Let $\Gamma^{\prime \prime}$ be a subgroup of $\mathrm{PSL}_{2}(\mathbb{C})$ where every element that fixes $\infty \in \partial \mathbb{U}_{\infty}^{3}$ is parabolic. Suppose there are positive constants $C_{1}$ and $C_{2}$ so that

(a) when

$$
\gamma=\left(\begin{array}{ll}
a & b \\
c & d
\end{array}\right) \in \Gamma^{\prime \prime}
$$

does not fix $\infty$, the entry c satisfies $|c| \geq C_{1}$;

(b) when $\gamma$ is a nontrivial element which fixes $\infty$, and is therefore of the form

$$
\left(\begin{array}{ll}
1 & b \\
0 & 1
\end{array}\right)
$$

the entry $b$ satisfies $|b| \geq C_{2}$.

Then there exists $\zeta \in \mathbb{M}^{3}$ such that, for every nontrivial element $\gamma \in \Gamma^{\prime \prime}$,

$$
\cosh d_{\mathbb{A}^{3}}(\gamma(\zeta), \zeta) \geq \frac{C_{1} C_{2}}{2} .
$$

Proof. Throughout the proof, we will be working in the upper-half space model. Let $\zeta=t \mathrm{j}$, where $t=\left(C_{2} / C_{1}\right)^{1 / 2}$ and $\mathrm{j}$ represents the vertical axis. It will be shown that $\zeta$ has the desired property. By well-known formulas [Matsuzaki and Taniguchi 1998], for

$$
\gamma=\left(\begin{array}{ll}
a & b \\
c & d
\end{array}\right)
$$

we have

$$
\gamma(\zeta)=\frac{b \bar{d}+a \bar{c} t^{2}+t \mathrm{j}}{|c \zeta+d|^{2}}
$$

and

$$
\begin{aligned}
\cosh d_{\mathbb{\boxplus}^{3}}(\gamma(\zeta), \zeta) & =\frac{|\gamma(\zeta)-\zeta|^{2}}{2 t^{2} /|c \zeta+d|^{2}}+1 \\
& =\frac{|\gamma(\zeta)-\zeta|^{2}|c \zeta+d|^{2}}{2 t^{2}}+1 \\
& =\frac{\left|\left(b \bar{d}+a \bar{c} t^{2}+t \mathrm{j}\right)-t \mathrm{j}\right| c \zeta+\left.\left.d\right|^{2}\right|^{2}}{2 t^{2}|c \zeta+d|^{2}}+1 \\
& =\frac{\left|b \bar{d}+a \bar{c} t^{2}+t \mathrm{j}\left(1-|c \zeta+d|^{2}\right)\right|^{2}}{2 t^{2}|c \zeta+d|^{2}}+1 \\
& =\frac{\left|b \bar{d}+a \bar{c} t^{2}\right|^{2}}{2 t^{2}|c \zeta+d|^{2}}+\frac{\left(1-|c \zeta+d|^{2}\right)^{2}}{2|c \zeta+d|^{2}}+1
\end{aligned}
$$

First, consider case (a). Since $c \zeta=c t \mathrm{j}$, we get $|c \zeta+d|^{2} \geq t^{2}|c|^{2}$. Clearly (5-1) is bigger than $|c \zeta+d|^{2} / 2$ so that it is bounded below by $\frac{1}{2} t^{2} C_{1}^{2}=C_{1} C_{2} / 2$, and $\cosh d_{\mathbb{\boxplus}^{3}}(\gamma(\zeta), \zeta)$ is as well. 
Second, consider case (b). In this case, we can rewrite $\cosh d_{\mathbb{\llbracket} 3}(\gamma(\zeta), \zeta)$ as

$$
\frac{b^{2}}{2 t^{2}}+1
$$

Obviously this is bounded below by

$$
\frac{C_{2}^{2}}{2 t^{2}}=\frac{C_{1} C_{2}}{2} .
$$

Now we apply the above lemma to congruence subgroups of $\Gamma^{\prime}$. First, let $\Gamma_{0}^{\prime}(I)$ be a Hecke-type congruence subgroup of $\Gamma^{\prime}$, and

$$
\gamma=\left(\begin{array}{ll}
a & b \\
c & d
\end{array}\right) \in \Gamma_{0}^{\prime}(I)
$$

where $I$ is an ideal of $O_{n}$. By the definition of the Hecke-type congruence subgroup, we have $c \in I$, so $\mathrm{N}(c)=|c|^{2} \geq \mathrm{N}(I)$ (when $c \neq 0$ ). Clearly $|b| \geq 1$ if $b \neq 0$. Furthermore, if $\gamma$ fixes $\infty$ (so $c=0$ ), $|a+d| \leq 2$, since $a$ and $d$ are conjugates of each other and both are units in the ring of integers in an imaginary quadratic number field. Therefore $\Gamma_{0}^{\prime}(I)$ satisfies all the conditions of the lemma. Thus there exists $\zeta \in \mathbb{\boxplus}^{3}$ such that $\cosh d_{\mathbb{H}^{3}}(\gamma(\zeta), \zeta)$ is bounded below by $\frac{1}{2} \mathrm{~N}(I)^{1 / 2}$ for all nontrivial $\gamma \in \Gamma_{0}^{\prime}(I)$. By a similar method, for a Hecke-type congruence subgroup $\Gamma_{1}^{\prime}(I)$ (respectively principal congruence subgroup $\Gamma^{\prime}(I)$ ), we get that $\cosh d_{\mathbb{1}^{3}}(\gamma(\zeta), \zeta)$ is bounded below by $\frac{1}{2} \mathrm{~N}(I)^{1 / 2}$ (respectively $\frac{1}{2} \mathrm{~N}(I)$ ) for all nontrivial $\gamma \in \Gamma_{1}^{\prime}(I)$ (respectively $\Gamma^{\prime}(I)$ ).

\section{Proof of Theorem 1.3}

Proof. If $P$ is a prime ideal of $\mathrm{O}_{n}$ and $M_{0}^{\prime}(P)$ is a congruence cover of $M^{\prime}$ induced by a Hecke-type congruence subgroup $\Gamma_{0}^{\prime}(P)$ of $\Gamma^{\prime}$, the upper injectivity radius $\overline{\operatorname{inj}}\left(M_{0}^{\prime}(P)\right)$ is bigger than

$$
\frac{1}{2} \cosh ^{-1}\left(\frac{1}{2} \mathrm{~N}(P)^{\frac{1}{2}}\right),
$$

by Lemma 5.1 and the observation at the end of Section 5. From

$$
\cosh ^{2} x=\frac{1}{2}(\cosh (2 x)+1)
$$

we get

$$
\cosh \left(\frac{1}{2} \cosh ^{-1}(x)\right)=\sqrt{\frac{x+1}{2}}>\sqrt{\frac{x}{2}}
$$

for $x \geq 1$. Combining this with Corollary 2.2 and (6-1), we have

$$
\text { Heegaard genus of } M_{0}^{\prime}(P) \geq \frac{\mathrm{N}(P)^{\frac{1}{4}}}{4} \text {. }
$$


Assuming $\mathrm{N}(I)$ is sufficiently large, the map in (2-8) is surjective, so, by the formula given in (2-9), the degree

$$
\left[\Gamma: \Gamma_{0}^{\prime}(P)\right]=\left[\Gamma: \Gamma^{\prime}\right]\left[\Gamma^{\prime}: \Gamma_{0}^{\prime}(P)\right]
$$

is equal to $d(\mathrm{~N}(P)+1)$, where $d=\left[\Gamma: \Gamma^{\prime}\right]$. Furthermore, for any $\epsilon>0$, we can easily check that

$$
\frac{\mathrm{N}(P)^{\frac{1}{4}}}{4} \geq(d(\mathrm{~N}(P)+1))^{\frac{1}{4}-\epsilon}
$$

holds for sufficiently large $\mathrm{N}(P)$. Thus we conclude that

$$
\text { Heegaard genus of } M_{0}^{\prime}(P) \geq\left(\left[\Gamma: \Gamma_{0}^{\prime}(P)\right]\right)^{\frac{1}{4}-\epsilon}
$$

with sufficiently large $\mathrm{N}(P)$.

For a given $\epsilon>0$, let $P_{1}, P_{2}, \ldots$ be a sequence of prime ideals of $\mathbb{O}_{n}$ such that each $M_{0}^{\prime}\left(P_{i}\right)$ satisfies the condition

$$
\frac{\mathrm{N}\left(P_{i}\right)^{\frac{1}{4}}}{4} \geq\left(d\left(\mathrm{~N}\left(P_{i}\right)+1\right)\right)^{\frac{1}{4}-\epsilon} .
$$

If we put $\Gamma_{i}=\Gamma_{0}^{\prime}\left(P_{1} \ldots P_{i}\right)$ and $M_{i}=$ cover of $M^{\prime}$ induced by $\Gamma_{i}$,

$$
\text { Heegaard genus of } M_{i} \geq \frac{\mathrm{N}\left(P_{1} \ldots P_{i}\right)^{\frac{1}{4}}}{4},
$$

by the same method we used to get (6-2). Since

$$
\frac{\mathrm{N}\left(P_{1} \ldots P_{i}\right)^{\frac{1}{4}}}{4} \geq\left(d\left(\mathrm{~N}\left(P_{1}\right)+1\right) \ldots\left(\mathrm{N}\left(P_{i}\right)+1\right)\right)^{\frac{1}{4}-\epsilon}
$$

by assumption, the inequality

$$
\text { Heegaard genus of } M_{i} \geq\left(d\left(\mathrm{~N}\left(P_{1}\right)+1\right) \ldots\left(\mathrm{N}\left(P_{i}\right)+1\right)\right)^{\frac{1}{4}-\epsilon}
$$

follows for all $i$. Now the sequence $\left\{\Gamma_{i}\right\}$ is the desired one for Theorem 1.3.

\section{Proof of Theorem 1.5}

Proof. Throughout the proof we suppose that $I$ is a square-free ideal that is not divisible by any of the prime ideals for which the map in (2-8) is not surjective. Under this assumption, we apply the explicit formulas (2-3), (2-9), and (2-10).

(i) As we saw in the proof of Theorem 1.3, the cover $M_{0}^{\prime}(I)$ contains a ball $B$ of radius greater than or equal to $\frac{1}{2} \cosh ^{-1}\left(\frac{1}{2} \mathrm{~N}(I)^{1 / 2}\right)>0$. Since

$$
\cosh ^{-1} \frac{x}{2}=\ln \frac{x+\sqrt{x^{2}-4}}{2}
$$


when $x \geq 2$ and $\cosh ^{-1}(x / 2) \geq 0$, we get

$$
\frac{1}{2} \cosh ^{-1}\left(\frac{1}{2} \mathrm{~N}(I)^{\frac{1}{2}}\right)=\frac{1}{2} \ln \frac{\mathrm{N}(I)^{\frac{1}{2}}+\sqrt{\mathrm{N}(I)-4}}{2}>\frac{1}{2} \ln \left(\mathrm{N}(I)^{\frac{1}{2}}-1\right)
$$

for $\mathrm{N}(I) \geq 4$. The volume of a hyperbolic ball of radius $r$ is

$$
\pi(\sinh (2 r)-2 r),
$$

so, for $r$ sufficiently large, it is bounded below by a constant multiple of $e^{2 r}$. This means that the volume of $B$ is greater than a constant multiple of $e^{\ln \left(\mathrm{N}(I)^{1 / 2}-1\right)}=$ $\mathrm{N}(I)^{1 / 2}-1$ with sufficiently large $\mathrm{N}(I)$. For convenience, we will simply assume that the volume of $B$ is bounded below by a constant multiple of $\mathrm{N}(I)^{1 / 2}$.

Let $I=P_{1} P_{2} \ldots P_{s}$ such that $P_{i}$ are distinct prime ideals and $\mathrm{N}\left(P_{i}\right)=p_{i}^{n_{i}}$, where $p_{i}$ are rational primes and $n_{i}=1$ or 2 depending on $P_{i}$. By the formula in (2-9), the degree of $M_{0}^{\prime}$ is equal to $\left(p_{1}^{n_{1}}+1\right)\left(p_{2}^{n_{2}}+1\right) \ldots\left(p_{s}^{n_{s}}+1\right)$. Now, for any $\epsilon>0$ and $c>0$, the inequality

$$
c\left(p_{1}^{n_{1}} p_{2}^{n_{2}} \ldots p_{s}^{n_{s}}\right)^{\frac{1}{2}}>\left(\left(p_{1}^{n_{1}}+1\right)\left(p_{2}^{n_{2}}+1\right) \ldots\left(p_{s}^{n_{s}}+1\right)\right)^{\frac{1}{2}-\epsilon}
$$

holds for sufficiently large $\mathrm{N}(I)=p_{1}^{n_{1}} p_{2}^{n_{2}} \ldots p_{s}^{n_{s}}$, because

$$
\frac{\left(p_{i}^{n_{i}}\right)^{\frac{1}{2}}}{\left(p_{i}^{n_{i}}+1\right)^{\frac{1}{2}-\epsilon}}=\left(p_{i}^{n_{i}}+1\right)^{\epsilon}\left(\frac{p_{i}^{n_{i}}}{p_{i}^{n_{i}}+1}\right)^{\frac{1}{2}}
$$

goes to infinity as $p_{i}$ increases. Part (i) is proved.

(ii) Because $\Gamma_{1}^{\prime}(I) \subset \Gamma_{0}^{\prime}(I)$, the cover $M_{1}^{\prime}(I)$ (induced by $\left.\Gamma_{1}^{\prime}(I)\right)$ also has a ball $B$ of radius at least $\frac{1}{2} \ln \left(\mathrm{N}(I)^{1 / 2}-1\right)$ for $\mathrm{N}(I) \geq 4$. As we checked in (i), the volume of this ball is greater than the constant multiple of $\mathrm{N}(I)^{1 / 2}$ for sufficiently large $\mathrm{N}(I)$. Since the degree of $\Gamma_{1}^{\prime}(I)$ is less than $\frac{1}{2} \mathrm{~N}(I)^{2}$, the statement in (ii) follows.

(iii) By the discussion at the end of Section 5,

$$
\cosh d_{\mathbb{\boxplus}^{3}}(\gamma(\zeta), \zeta) \geq \frac{1}{2} \mathrm{~N}(I)
$$

for a principal congruence subgroup $\Gamma^{\prime}(I)$ and any nontrivial $\gamma \in \Gamma^{\prime}(I)$. Following the same way as in (i), we can prove that the cover $M^{\prime}(I)$ (induced by $\Gamma^{\prime}(I)$ ) contains a ball $B$ of volume bounded below by a constant multiple of $\mathrm{N}(I)$. Because the degree of $M^{\prime}(I)$ is less than $\frac{1}{2} \mathrm{~N}(I)^{3}$, we arrive at the desired conclusion.

\section{Final comments}

8A. In the proof of Theorem 1.2, we picked the prime number $p$ using Lemma 3.2. But we can choose a different prime directly from Lemma 3.1. By Lemma 3.1 (with the same notations as in Section 3), for every $\omega \in \Gamma$ of length less than or equal to $l$, there exist $\alpha, \beta \in \mathbb{O}_{K}$ such that $\operatorname{tr} \hat{\omega}=\alpha / \beta$ and $\mathrm{N}(\alpha \pm 2 \beta) \leq C_{3}^{l}$. If we select a prime 
$p_{1}$ which is bigger than $C_{3}^{l}$ and smaller than $2 C_{3}^{l}$, then, for a prime ideal factor $P_{1}$ of $p_{1} \mathcal{O}_{K}$, a Hecke-type congruence subgroup $\Gamma_{1}\left(P_{1}\right)$ does not contain any element of length less than or equal to $l$, because $\mathrm{N}\left(P_{1}\right) \nmid \prod_{i=1}^{r(l)}\left|\mathrm{N}\left(\alpha_{i}-2 \beta_{i}\right)\right|\left|\mathrm{N}\left(\alpha_{i}+2 \beta_{i}\right)\right|$ and the same reasoning as in the proof of Claim 3.3. Now, applying Chebotarev's density theorem, pick a prime number $p_{1}^{\prime}$ such that $p_{1}^{\prime}$ splits completely in $\mathbb{O}_{K}$ and $p_{1}<p_{1}^{\prime}<3 p_{1}$. Since $p_{1}^{\prime}$ also satisfies $p_{1}^{\prime} \nmid \prod_{i=1}^{r(l)}\left|\mathrm{N}\left(\alpha_{i}-2 \beta_{i}\right)\right|\left|\mathrm{N}\left(\alpha_{i}+2 \beta_{i}\right)\right|$ for any prime factor $P_{1}^{\prime}$ of $p_{1}^{\prime} \mathrm{O}_{K}$, a Hecke-type congruence subgroup $\Gamma_{1}\left(P_{1}^{\prime}\right)$ does not contain any element of length less than or equal to $l$. Hence we get

Heegaard genus of the cover corresponding to $\Gamma_{1}\left(P_{1}^{\prime}\right) \geq \frac{e^{l / 2}}{4}$, and, from (2-10),

$$
\text { Degree of } \Gamma_{1}\left(P_{1}^{\prime}\right)<\frac{1}{2}\left(p_{1}^{\prime}\right)^{2}<\frac{1}{2}\left(6 C_{3}^{l}\right)^{2} \text {, }
$$

because $p_{1}^{\prime}<3 p_{1}, p_{1}<2 C_{3}^{c l}$, and $\mathrm{N}\left(P_{1}^{\prime}\right)=p_{1}^{\prime}$.

However the problem in this case is that we don't know exactly how big the constant $C_{3}$ is. In particular, the constant $C_{3}$ strongly depends on $\Gamma$. Thus the result coming from the above line of reasoning is weaker than the result obtained using Lemma 3.2, which is universally independent of $\Gamma$.

8B. The reader might wonder why we chose to work with the Hecke-type subgroup $\Gamma_{1}(P)$ instead of the principal congruence subgroup $\Gamma(P)$ in Theorem 1.2. In fact, using $\Gamma(P)$ gives Degree ${ }^{1 / 12-\epsilon}$ as a lower bound of the Heegaard genus of the induced cover. Although, for a given $l, \Gamma(P)$ allows us to take a smaller upper bound on $\mathrm{N}(P)$, it doesn't offset the increase of the degree. More precisely, if a hyperbolic element $\omega$ is contained in $\Gamma(P)$, we have $\operatorname{tr} \hat{\omega} \equiv \pm 2 \in P^{2}$ (compare to the case $\operatorname{tr} \hat{\omega} \equiv \pm 2 \in P$ when $\left.\omega \in \Gamma_{1}(P)\right)$ so that we can pick a rational prime $p$ with a loosened condition $p^{2} \nmid \prod_{i=1}^{r(l)}\left|\mathrm{N}\left(\alpha_{i}-2 \beta_{i}\right)\right|\left|\mathrm{N}\left(\alpha_{i}+2 \beta_{i}\right)\right|$, rather than $p \nmid \prod_{i=1}^{r(l)}\left|\mathrm{N}\left(\alpha_{i}-2 \beta_{i}\right)\right|\left|\mathrm{N}\left(\alpha_{i}+2 \beta_{i}\right)\right|$, in the argument after Claim 3.3. By slightly changing the proof of Corollary 2.5, it is not difficult to see that, for sufficiently large $x$, there exists a prime number $p \in S_{2}$ such that $p^{2} \nmid x$ and $p<n \log x$ (compare to the case $2 n \log x$ of $\Gamma_{1}(P)$ ). But, as we can check from the proof of Theorem 1.2, $n \log x$ doesn't improve the result that much. On the contrary, since the degree of $\Gamma(P)$ has a cube power of $\mathrm{N}(P)$ as one of its term, the lower bound of the Heegaard genus of the cover decreases from Degree ${ }^{1 / 8-\epsilon}$ to Degree ${ }^{1 / 12-\epsilon}$.

8C. Now we heuristically explore the limits of Theorem 2.1. For a given closed hyperbolic 3-manifold $M$, first find the maximum upper injectivity radius of $M$. If $r=\overline{\operatorname{inj}}(M)$ and $B(r)$ is a hyperbolic ball of radius $r$ embedded in $M$, then, by assuming

$$
\operatorname{vol} M=\operatorname{vol} B(r),
$$


one can calculate the largest possible value of $r$. Since

$$
\operatorname{vol} M=\operatorname{vol} B(r) \approx \pi e^{2 r},
$$

for sufficiently large values of vol $M$, it follows that

$$
r \approx \frac{1}{2} \ln \frac{\operatorname{vol} M}{\pi}
$$

For the convenience of calculation, we simply assume $r=\frac{1}{2} \ln (\operatorname{vol} M / \pi)$. Applying this value of $r$ to Theorem 2.1, we have

$$
\text { Heegaard genus of } M \geq \frac{1}{4 \sqrt{\pi}}(\operatorname{vol} M)^{\frac{1}{2}} \text {. }
$$

In conclusion, $\frac{1}{2}$ is the largest value for the exponent of vol $M$ in Theorem 1.2 that we can get using Theorem 2.1. Recalling Theorem 1.1, we can say that Theorem 2.1 would have to be improved substantially in the arithmetic case to give an alternative proof of Theorem 1.1.

\section{Acknowledgement}

I am extremely grateful to my advisor Nathan Dunfield for all his help, without which this paper wouldn't have been possible. I also thank Paul Pollack and Jonah Sinick for many helpful comments. Lastly, I thank the anonymous referee for careful reading and corrections.

\section{References}

[Bachman et al. 2004] D. Bachman, D. Cooper, and M. E. White, "Large embedded balls and Heegaard genus in negative curvature", Algebr. Geom. Topol. 4 (2004), 31-47. MR 2004m:57029 Zbl 1056.57014

[Bateman and Diamond 2004] P. T. Bateman and H. G. Diamond, Analytic number theory: an introductory course, World Scientific, Hackensack, NJ, 2004. MR 2005h:11208 Zbl 1074.11001

[Lackenby 2006] M. Lackenby, "Heegaard splittings, the virtually Haken conjecture and property ( $\tau$ )", Invent. Math. 164:2 (2006), 317-359. MR 2007c:57030 Zbl 1110.57015

[Lackenby 2011] M. Lackenby, "Finite covering spaces of 3-manifolds", pp. 1042-1070 in Proceedings of the International Congress of Mathematicians, II (Hyderabad, 2010), edited by R. Bhatia et al., Hindustan Book Agency, New Delhi, 2011. MR 2827831 Zbl 1233.57012

[Long and Reid 1998] D. D. Long and A. W. Reid, "Simple quotients of hyperbolic 3-manifold groups”, Proc. Amer. Math. Soc. 126:3 (1998), 877-880. MR 98e:57022 Zbl 0891.57012

[Long et al. 2008] D. D. Long, A. Lubotzky, and A. W. Reid, "Heegaard genus and property $\tau$ for hyperbolic 3-manifolds", J. Topol. 1:1 (2008), 152-158. MR 2008j:57036 Zbl 1158.57018

[Lubotzky 1994] A. Lubotzky, Discrete groups, expanding graphs and invariant measures, Progress in Mathematics 125, Birkhäuser, Basel, 1994. MR 96g:22018 Zbl 0826.22012 
[Maclachlan and Reid 2003] C. Maclachlan and A. W. Reid, The arithmetic of hyperbolic 3-manifolds, Graduate Texts in Mathematics 219, Springer, New York, 2003. MR 2004i:57021 Zbl 1025. 57001

[Margulis 1969] G. A. Margulis, "Applications of ergodic theory to the investigation of manifolds of negative curvature”, Funkcional. Anal. i Priložen. 3:4 (1969), 89-90. In Russian; translated in Funct. Anal. Appl. 03 (1969), 335-336. MR 41 \#2582 Zbl 0207.20305

[Marklof 1996] J. Marklof, "On multiplicities in length spectra of arithmetic hyperbolic threeorbifolds", Nonlinearity 9:2 (1996), 517-536. MR 97d:11086 Zbl 1043.11526

[Matsuzaki and Taniguchi 1998] K. Matsuzaki and M. Taniguchi, Hyperbolic manifolds and Kleinian groups, Clarendon, New York, 1998. MR 99g:30055 Zbl 0892.30035

[Milnor 1968] J. Milnor, "A note on curvature and fundamental group", J. Differential Geometry 2 (1968), 1-7. MR 38 \#636 Zbl 0162.25401

[Miyake 1989] T. Miyake, Modular forms, Springer, Berlin, 1989. MR 90m:11062 Zbl 0701.11014

[Narkiewicz 2004] W. Narkiewicz, Elementary and analytic theory of algebraic numbers, 3rd ed., Springer, Berlin, 2004. MR 2005c:11131 Zbl 1159.11039

[Rubinstein 2005] J. H. Rubinstein, "Minimal surfaces in geometric 3-manifolds", pp. 725-746 in Global theory of minimal surfaces, edited by D. Hoffman, Clay Math. Proc. 2, Amer. Math. Soc., Providence, RI, 2005. MR 2006g:57038 Zbl 1119.53042

[Weisfeiler 1984] B. Weisfeiler, "Strong approximation for Zariski-dense subgroups of semisimple algebraic groups", Ann. of Math. (2) 120:2 (1984), 271-315. MR 86m:20053 Zbl 0568.14025

[Yeung 1994] S.-K. Yeung, "Betti numbers on a tower of coverings", Duke Math. J. 73:1 (1994), 201-226. MR 95e:58182 Zbl 0818.22007

Received July 31, 2011.

\section{BOGWANG JEON}

DEPARTMENT OF MATHEMATICS

UNIVERSITY OF ILLINOIS AT URBANA-CHAMPAIGN

1409 W. GREEN STREET

URBANA, ILLINOIS 61801

UNITED STATES

jeon14@illinois.edu 


\title{
PACIFIC JOURNAL OF MATHEMATICS
}

\author{
http://pacificmath.org \\ Founded in 1951 by \\ E. F. Beckenbach (1906-1982) and F. Wolf (1904-1989)
}

\section{EDITORS}

V. S. Varadarajan (Managing Editor)

Department of Mathematics

University of California

Los Angeles, CA 90095-1555

pacific@math.ucla.edu

Vyjayanthi Chari

Department of Mathematics

University of California

Riverside, CA 92521-0135

chari@math.ucr.edu

\section{Robert Finn}

Department of Mathematics Stanford University

Stanford, CA 94305-2125

finn@math.stanford.edu

Kefeng Liu

Department of Mathematics

University of California

Los Angeles, CA 90095-1555

liu@math.ucla.edu
Darren Long

Department of Mathematics

University of California

Santa Barbara, CA 93106-3080

long@math.ucsb.edu

Jiang-Hua Lu

Department of Mathematics

The University of Hong Kong

Pokfulam Rd., Hong Kong jhlu@maths.hku.hk

Alexander Merkurjev

Department of Mathematics

University of California

Los Angeles, CA 90095-1555

merkurev@math.ucla.edu
Sorin Popa

Department of Mathematics University of California

Los Angeles, CA 90095-1555 popa@math.ucla.edu

Jie Qing

Department of Mathematics

University of California

Santa Cruz, CA 95064

qing@cats.ucsc.edu

Jonathan Rogawski

Department of Mathematics

University of California

Los Angeles, CA 90095-1555

jonr@math.ucla.edu

\section{PRODUCTION}

pacific@math.berkeley.edu

\section{SUPPORTING INSTITUTIONS}

ACADEMIA SINICA, TAIPEI

CALIFORNIA INST. OF TECHNOLOGY INST. DE MATEMÁTICA PURA E APLICADA KEIO UNIVERSITY

MATH. SCIENCES RESEARCH INSTITUTE NEW MEXICO STATE UNIV.

OREGON STATE UNIV.

\author{
STANFORD UNIVERSITY \\ UNIV. OF BRITISH COLUMBIA \\ UNIV. OF CALIFORNIA, BERKELEY \\ UNIV. OF CALIFORNIA, DAVIS \\ UNIV. OF CALIFORNIA, LOS ANGELES \\ UNIV. OF CALIFORNIA, RIVERSIDE \\ UNIV. OF CALIFORNIA, SAN DIEGO \\ UNIV. OF CALIF., SANTA BARBARA
}

\author{
UNIV. OF CALIF., SANTA CRUZ \\ UNIV. OF MONTANA \\ UNIV. OF OREGON \\ UNIV. OF SOUTHERN CALIFORNIA \\ UNIV. OF UTAH \\ UNIV. OF WASHINGTON \\ WASHINGTON STATE UNIVERSITY
}

These supporting institutions contribute to the cost of publication of this Journal, but they are not owners or publishers and have no responsibility for its contents or policies.

See inside back cover or pacificmath.org for submission instructions.

The subscription price for 2012 is US \$420/year for the electronic version, and \$485/year for print and electronic.

Subscriptions, requests for back issues from the last three years and changes of subscribers address should be sent to Pacific Journal of Mathematics, P.O. Box 4163, Berkeley, CA 94704-0163, U.S.A. Prior back issues are obtainable from Periodicals Service Company, 11 Main Street, Germantown, NY 12526-5635. The Pacific Journal of Mathematics is indexed by Mathematical Reviews, Zentralblatt MATH, PASCAL CNRS Index, Referativnyi Zhurnal, Current Mathematical Publications and the Science Citation Index.

The Pacific Journal of Mathematics (ISSN 0030-8730) at the University of California, c/o Department of Mathematics, 969 Evans Hall, Berkeley, CA 94720-3840, is published monthly except July and August. Periodical rate postage paid at Berkeley, CA 94704, and additional mailing offices. POSTMASTER: send address changes to Pacific Journal of Mathematics, P.O. Box 4163, Berkeley, CA 94704-0163.

PJM peer review and production are managed by EditFLOW ${ }^{\mathrm{TM}}$ from Mathematical Sciences Publishers.

PUBLISHED BY PACIFIC JOURNAL OF MATHEMATICS

at the University of California, Berkeley 94720-3840

A NON-PROFIT CORPORATION

Typeset in LATEX

Copyright $(02012$ by Pacific Journal of Mathematics 


\section{PACIFIC JOURNAL OF MATHEMATICS}

Volume $258 \quad$ No. $1 \quad$ July 2012

On the complexity of sails

LUKAS BRANTNER

Construction of Lagrangian submanifolds in $\mathbb{C P}^{n}$

QING Chen, SEN Hu and XiaOweI XU

Semisimple tunnels

SANGBUM CHO and DARRYL MCCULLOUGH

Degenerate two-boundary centralizer algebras

\section{ZAJJ DAUGHERTY}

Heegaard genera in congruence towers of hyperbolic 3-manifolds

BOGWANG JEON

The Heisenberg ultrahyperbolic equation: The basic solutions as distributions

ANTHONY C. KABLE

Rational Seifert surfaces in Seifert fibered spaces

JOAN E. LiCATA and JoshuA M. SABLOFF

Delaunay cells for arrangements of flats in hyperbolic space 